\title{
Peri-implantitis Update: Risk Indicators, Diagnosis, and Treatment
}

\author{
Dinesh Rokaya ${ }^{1,2}$ Viritpon Srimaneepong ${ }^{3}$ Wichaya Wisitrasameewon ${ }^{4}$ Manoj Humagain ${ }^{5}$ \\ Pasutha Thunyakitpisal ${ }^{2,6}$
}

${ }^{1}$ International College of Dentistry, Walailak University, Bangkok, Thailand

${ }^{2}$ Research Unit of Herbal Medicine, Biomaterials and Materials for Dental Treatment, Faculty of Dentistry, Chulalongkorn University, Bangkok, Thailand

${ }^{3}$ Department of Prosthodontics, Faculty of Dentistry, Chulalongkorn

University, Bangkok, Thailand

${ }^{4}$ Department of Periodontology, Faculty of Dentistry,

Chulalongkorn University, Bangkok, Thailand

${ }^{5}$ Department of Periodontics, Kathmandu University School of

Medical Sciences, Dhulikhel, Kavre, Nepal

${ }^{6}$ Department of Anatomy, Faculty of Dentistry, Chulalongkorn

University, Bangkok, Thailand

Eur J Dent:2020;14:672-682
Address for correspondence Viritpon Srimaneepong, DDS, MDSc, PhD, Department of Prosthodontics, Faculty of Dentistry, Chulalongkorn University, Bangkok 10330, Thailand (e-mail:viritpon.s@chula.ac.th).

\begin{abstract}
Keywords

- dental implants

- peri-implantitis

- implant complications

- decontamination

- anti-infective agents

- periodontal

debridement

- bone regeneration

Despite the success rates of dental implants, peri-implantitis presents as the most common complication in implant dentistry. This review discusses various factors associated with peri-implantitis and various available treatments, highlighting their advantages and disadvantages. Relevant articles on peri-implantitis published in English were reviewed from August 2010 to April 2020 in MEDLINE/PubMed, Scopus, and ScienceDirect. The identified risk indicators of peri-implant diseases are plaque, smoking, history of periodontitis, surface roughness, residual cement, emergence angle $>30$ degrees, radiation therapy, keratinized tissue width, and function time of the implant, sex, and diabetes. Peri-implantitis treatments can be divided into nonsurgical (mechanical, antiseptic, and antibiotics), surface decontamination (chemical and laser), and surgical (air powder abrasive, resective, and regenerative). However, mechanical debridement alone may fail to eliminate the causative bacteria, and this treatment should be combined with other treatments (antiseptics and surgical treatment). Surface decontamination using chemical agents may be used as an adjuvant treatment; however, the definitive clinical benefit is yet not proven. Laser treatment may result in a short-term decrease in periodontal pocket depth, while air powder abrasive is effective in cleaning a previously contaminated implant surface. Surgical elimination of a pocket, bone recontouring and plaque control are also effective for treating peri-implantitis. The current evidence indicates that regenerative approaches to treat peri-implant defects are unpredictable.
\end{abstract}

\section{Introduction}

The dental implant has revolutionized oral rehabilitation and become a part of routine treatment in prosthetic rehabilitation. ${ }^{1}$ There has been marked advancement in implant design, materials used, and surgical protocols. A high implant survival rate (94.6\%) has been reported over a 13.4-year follow-up. ${ }^{2}$ Approximately $90 \%$ of patients who received an implant were satisfied with their chewing ability and 
accessibility for plaque control at the implant sites. Despite high long-term survival rates, complications due to peri-implant diseases are frequent and, in severe cases, result in the loss of the implants and their prostheses.

Peri-implant diseases affect the tissues around the implants and have an inflammatory origin. They present in the following two forms: peri-implant mucositis and peri-implantitis. In peri-implant mucositis, the inflammation is restricted to the peri-implant tissue without a marginal bone loss ( - Fig. 1). Peri-implant mucositis is reversible through early treatment by eliminating the etiology. Periimplantitis is an inflammation of the peri-implant mucosa accompanied by marginal bone loss ( $\boldsymbol{- \text { Fig. }}$ 2). Peri-implant mucositis and peri-implantitis have a high-prevalence. The mean implant-based and subject-based peri-implant mucositis prevalence was $29.48 \%$ and $46.83 \%$, respectively, and the mean implant-based and subject-based peri-implantitis prevalence was $9.25 \%$ and $19.83 \%$, respectively. ${ }^{3}$

\section{Method}

This article discusses various factors associated with peri-implantitis and various treatments, highlighting their advantages and disadvantages. Relevant articles on periimplantitis published in English were reviewed from August 2010 to April 2020 in MEDLINE/PubMed, Scopus, and ScienceDirect.

\section{Etiologies and Risk Indicators of Peri-implant Diseases}

Similar to periodontal diseases, the prime causative factor for peri-implant diseases is dental plaque. ${ }^{4}$ Periodontal health is influenced by various factors such as oral hygiene, genetic and epigenetic factors, systemic health, and nutrition., ${ }^{5,6}$ Peri-implantitis and periodontitis lesions both harbor Gramnegative anaerobic bacteria compared with healthy sites. However, peri-implantitis has higher microbial diversity than periodontitis. ${ }^{7}$ Moreover, peri-implantitis is penetrated predominantly by inflammatory cells, B-lymphocytes and plasma cells, and frequently lacks a protective tissue layer over the bone, which is typically present in periodontitis. Histologically, peri-implantitis lesions were twice as large and had more blood vessels and the infiltrate in the connective tissue compared with perodontitis. ${ }^{8}$ Peri-implantitis demonstrated a 97\% higher matrix metalloproteinases (MMP) level, such as MMP-8, which was only $78 \%$ greater in chronic periodontitis compared with healthy gingiva. ${ }^{9}$ Furthermore, peri-implantitis tissue contains extracellular matrix antibodies. ${ }^{10}$ The disease progression rate is faster in peri-implantitis, which generates a faster and more severe loss of bone compared with periodontal disease. A nonlinear form of progressive bone destruction occurs over time in peri-implantitis, which maybe because of the differences in microorganisms at the implant sites, the host's defense mechanism, and absence of a periodontal ligament. ${ }^{11,12}$
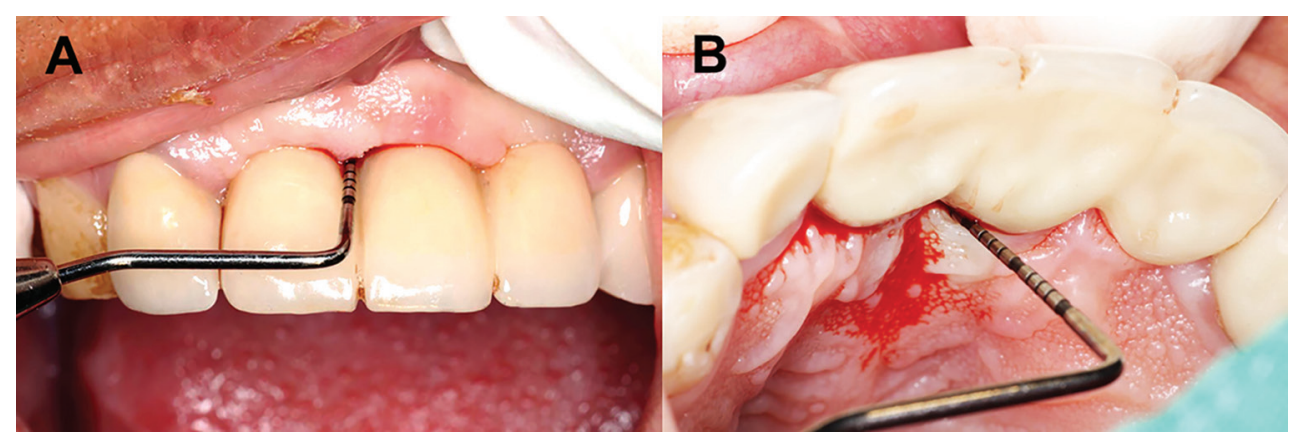

Fig. 1 Peri-implant mucositis demonstrating soft-tissue inflammation.

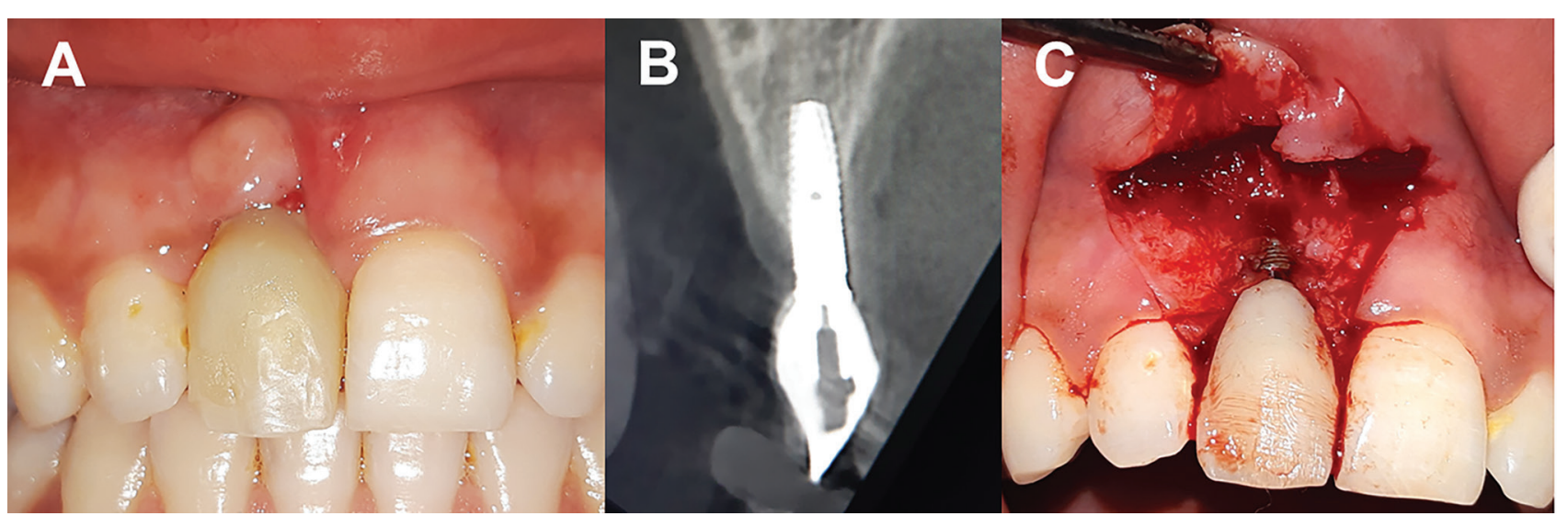

Fig. 2 Peri-implantitis: (A) clinical photograph, (B) radiograph showing bone loss around an implant, and (C) clinical photograph showing buccal bone loss. 
Risk indicators are referred to as factors associated with peri-implant diseases. However, to identify true risk factors, prospective studies are needed because there are currently few such studies. Thus, the term risk indicators were used in most studies. The identified risk indicators of peri-implant diseases are plaque, smoking, history of periodontitis, implant design and surface roughness of the transmucosal portion, residual cement, emergence angle >30 degrees, radiation therapy, width of the keratinized tissue and function time of the implant, sex, and diabetes. ${ }^{13-17}$ In addition to these, other factors associated with peri-implantitis are occlusal overload, ${ }^{18}$ history of implant failure, patients' parafunctional habits, and improper implant position. ${ }^{19-21}$ Periimplant mucositis is associated with an increased risk of becoming peri-implantitis. However, there are limited data available to support any systemic conditions as risk indicators for peri-implant mucositis. Limited evidence has shown the correlation of peri-implant diseases with alcohol consumption. Systemic diseases such as scleroderma, ectodermal dysplasia, lichen planus, osteoporosis, rheumatoid arthritis, and Sjögren's syndrome may have negative effects on peri-implantitis and implant success. ${ }^{15,22}$ To confirm these findings, additional detailed studies are needed. Genetic traits may be correlated with peri-implant diseases; however, the results are conflicting and limited.

Prosthetic restorations are associated with peri-implant diseases. There are three types of implant-abutment connections: platform-switched, butt-joint, and no interface. ${ }^{19}$ Bone loss of approximately 1.5 to $2.0 \mathrm{~mm}$ may occur with buttjoint connections due to the micro gap, which is wide enough for bacterial penetration and colonization. Although platform switching prevents or reduces marginal bone loss, ${ }^{20}$ contaminated connections can cause peri-implantitis and implant failure over time. Moreover, a convex restoration profile creates an additional risk for bone-level implants. ${ }^{15}$

Retained cement left on the implant surface after crown cementation is a potential risk for peri-implantitis because retained cement has an adverse effect on peri-implant tissues (-Fig. 3). ${ }^{14,23}$ The position of the implant, such as too apical or angled, and an overcontoured crown, negatively affect the accessibility for removing excess cement from the subgingival space. An implant splinted to both a mesial and distal adjacent implant has a higher risk of peri-implantitis. ${ }^{24}$ Cement causes roughness, favors bacterial attachment and foreign body reactions, and results in peri-implantitis. Removing the cement results in the resolution of inflammation in a few days to weeks and can be done using a closed procedure (dental endoscope) or open surgical flap procedure. Hence, to reduce the risk of peri-implant disease associated with excess cement, it is recommended that the crown margin is at the level of the mucosal margin, providing sufficient access and soft-tissue maturation, and early follow-up evaluation after restoration placement should be done. ${ }^{14}$

Mechanical stress beyond the threshold (occlusal overloading) is also considered a major cause of screw loosening or implant body fracture or other components. Increased mechanical stress can result in a greater concentration of force on the cervical part of the implant. ${ }^{25,26}$ Furthermore, overloading and increased loading time cause fatigue microdamage that results in bone resorption which may, in turn, progress to peri-implantitis. ${ }^{27}$ Moreover, increased bone remodeling around the implant is seen when the implant is subjected to high-loading forces. Mutually protected occlusal schemes and favorable contacts, avoiding cantilevers, narrowing the occlusal table, increasing implant number when replacing teeth, decreasing cusp inclines, increasing contact points, and eliminating parafunctional habits can reduce peri-implantitis. $^{28}$

\section{Diagnosis of Peri-implant Diseases}

Clinical and radiograph examination is necessary to diagnose peri-implant health and diseases. Therefore, a baseline clinical and radiographic examination is required when placing an implant. This information serves as a reference for evaluating physical or pathological changes in peri-implant tissues over time.

Generally, a healthy peri-implant tissue shows no sign of inflammation, bleeding on probing (BOP), or increase in probing depth (PD) compared with the initial or baseline examination. The diagnostic definition of peri-implant health is based on the following criteria: (1) absence of peri-implant signs of soft tissue inflammation (redness, swelling, or profuse bleeding on probing), and (2) the absence of additional

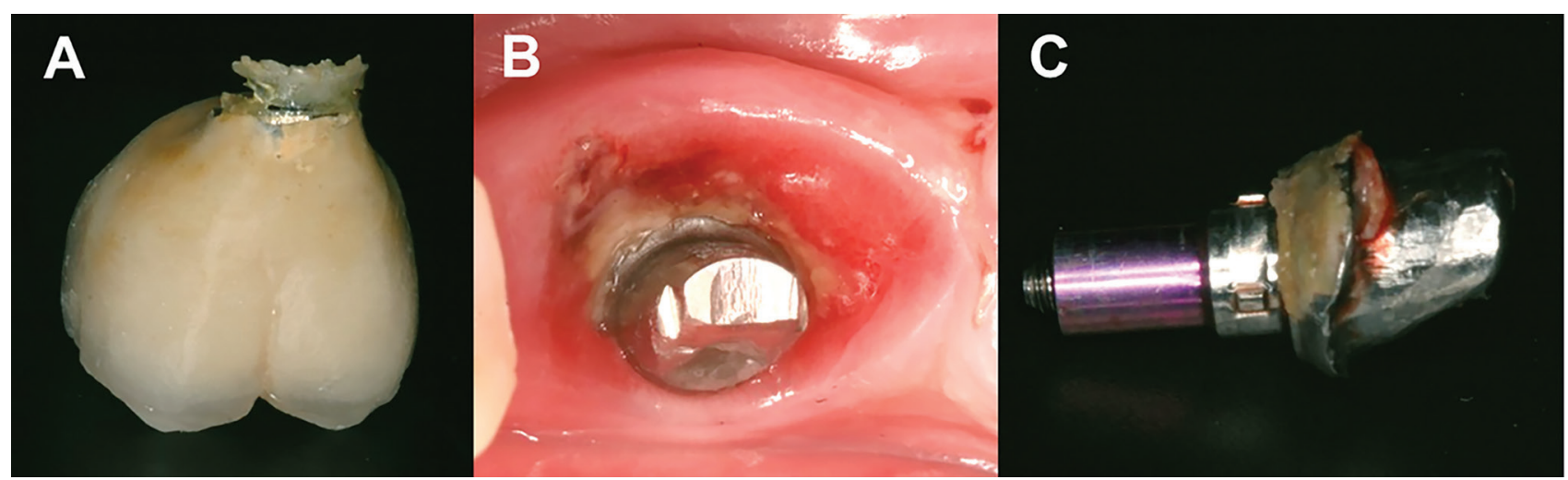

Fig. 3 Retained cement results in peri-implantitis: (A) retained cement at the crown margin and excess cement in the peri-implant inflamed tissue, and (B) excess cement around the abutment. Reproduced from Ref. ${ }^{23}$ with permission from John Wiley \& Sons A/S. 
bone loss following initial healing..$^{29}$ An increased PD might indicate attachment loss and supporting bone loss. ${ }^{17}$ The correct diagnosis is crucial to develop an appropriate treatment plan, leading to the successful treatment of peri-implant diseases.

According to the World Workshop on the Classification of Periodontal and Peri-Implant Diseases and Conditions (2018), ${ }^{29}$ peri-implant mucositis can be diagnosed based on the following criteria: (1) presence of peri-implant signs of inflammation (redness, swelling, line or bleeding within 30 second after probing), combined with (2) no additional bone loss following initial healing. Peri-implantitis can be diagnosed clinically based on the following criteria: (1) presence of peri-implant signs of inflammation, (2) radiographic evidence of bone loss following initial healing, and (3) increased probing depth compared with the probing depth after placing the prosthetic reconstruction. In the absence of previous radiographs, a radiographic bone level $\geq 3 \mathrm{~mm}$ in combination with BOP and PD $\geq 6 \mathrm{~mm}$ is indicative of peri-implantitis.

\section{Peri-implantitis Treatment}

Peri-implant diseases share similar clinical features and etiologies to periodontal diseases; thus, similar treatment approaches have been adopted to manage them. However, treatment outcomes vary. Mucositis treatment is more predictable; in contrast, peri-implantitis treatment is difficult, and the outcome varies. Therefore, supportive therapy at the initial stage reduces the risk of the onset of peri-implantitis. ${ }^{30,31}$ Various treatments for peri-implant diseases are presented in -Fig. 4. Peri-implant maintenance therapy or supportive therapy (SPT) increases the implant survival rate. ${ }^{32}$

An appropriate management strategy should consider local and systemic factors. ${ }^{33}$ Long-term supportive therapy is recommended for peri-implant diseases. Its main goals are infection control, prevent disease progression, and restore the lost bone. This protocol underscores the routine follow-up of the implant patient with periodic assessment of plaque and calculus, BOP, PD, and radiological evaluation for bone loss. These parameters indicate disease severity and extent. According to the CIST protocol, depending on the clinical and radiographic findings, different treatment approaches are indicated.

\section{Nonsurgical Treatment}

The different nonsurgical therapy of peri-implant disease comprises mechanical, chemical, antibiotics, lasers, and oral hygiene instruction.

\section{Mechanical Methods}

Mechanical debridement reduces inflammation by removing microbial plaque on the implant surface. Mechanical instruments for plaque removal include plastic curettes, ultrasonic scalers with a metal tip, metal curettes, air abrasive, and metallic (titanium) brushes (-Fig. 5). ${ }^{34}$ Piezoelectric scalers and hand instruments are also effective in reducing BOP score, plaque index, and PD. Ultrasonic scalers with metal tips and metal curettes can eliminate surface material down to $0.83 \mu \mathrm{m}$ in size and efficiently remove bacteria. ${ }^{35}$ However, these must be used carefully because they may create scratches on the implant surfaces if used improperly. ${ }^{36}$ Although plastic curettes are also available, they may not incompletely remove the debris or biofilm. Mechanical plaque removal methods can be combined with antibiotics or surgical methods for a better outcome. In a randomized trial, Toma et $\mathrm{al}^{34}$ compared three mechanical treatments (air-abrasive device, titanium brush, and plastic curettes,) for peri-implantitis. They found that the air-polishing device and titanium brush were more efficient than the others; however, the success was low.

Persson et $\mathrm{al}^{37}$ performed a single-blinded, longitudinal, randomized study to assess the effects of mechanical debridement on the peri-implant microbiota in peri-implantitis lesions wherein they tested 79 different microorganisms. They found no microbiological differences for implants treated with the ultrasonic device. Inconsistent changes occurred following the first week. No microbiological differences were found for any species or between treatment

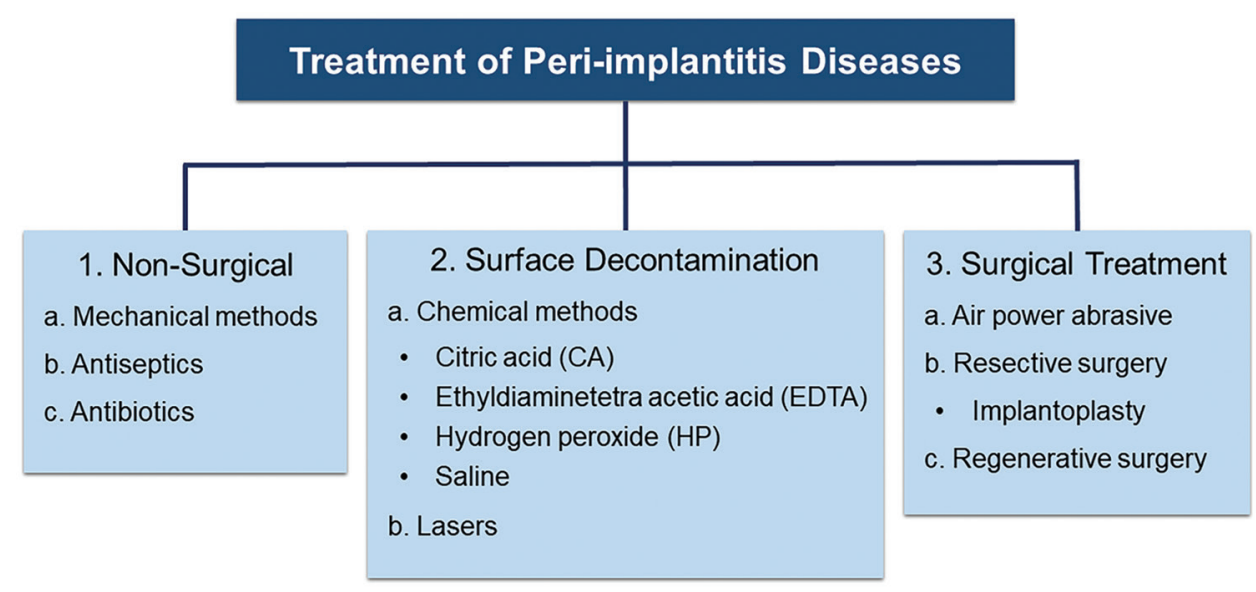

Fig. 4 Various treatments for peri-implant diseases. 


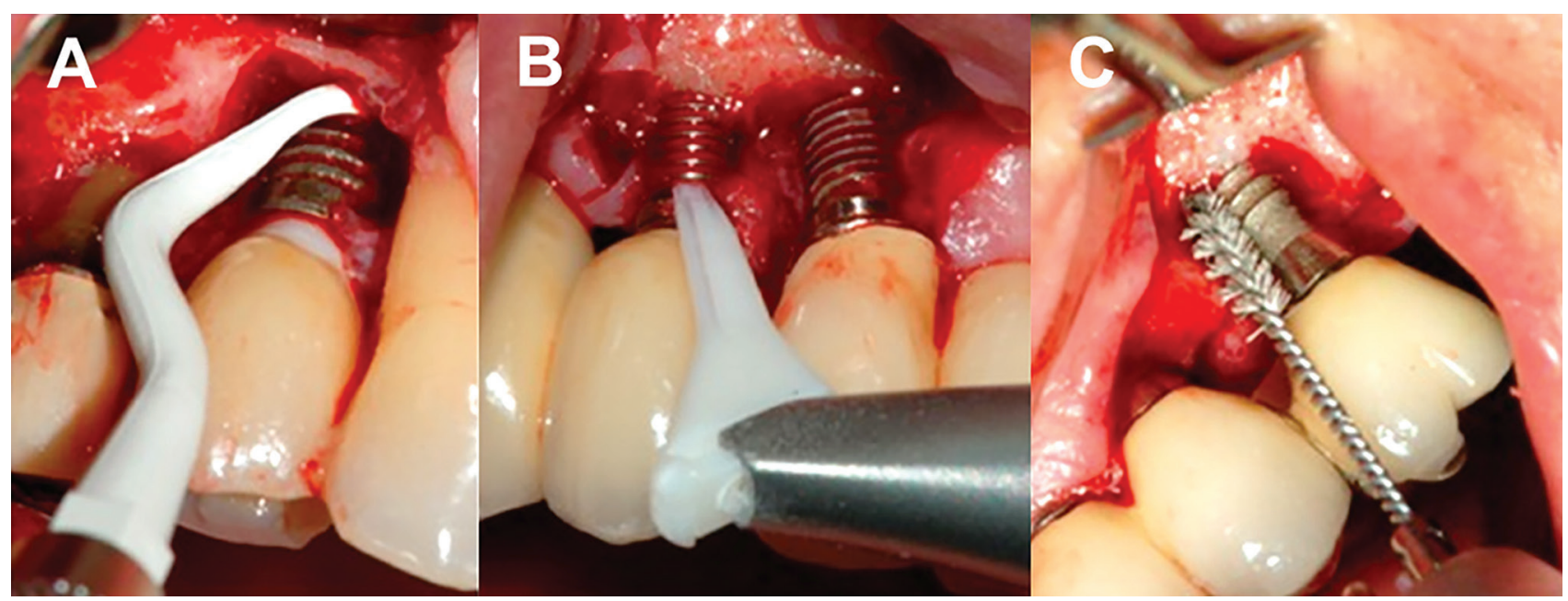

Fig. 5 Mechanical treatments of peri-implantitis: (A) plastic curettes, (B) air abrasive, and (C) metallic brush. Reproduced from Ref. ${ }^{34}$ under the Creative Commons Attribution License (CC BY 4.0) from Frontiers Media S.A.

study methods in peri-implantitis between the baseline and 6 -month samples. They concluded that both methods failed to eliminate or reduce bacterial counts in peri-implantitis. Moreover, the adjunctive use of antimicrobial mouth rinses enhances the outcome of mechanical therapy in peri-implantitis. ${ }^{38}$ Furthermore, surgical procedures (open flap debridement) increase the effectiveness of mechanical treatment of peri-implantitis. ${ }^{39}$ Hence, mechanical debridement alone may fail to eliminate bacteria and this treatment should be combined with other treatments (antiseptics and surgical treatment).

\section{Antiseptics}

Antiseptics are mainly indicated for reducing bacterial counts and can be used in the form of local irrigation. Chlorhexidine (CHX) gluconate is commonly used in periodontitis and peri-implant diseases. CHX retards bacterial colonization and its $0.12 \%$ concentration effectively reduces peri-implantitis disease. ${ }^{40,41}$ Hence, CHX is useful as an antiseptic agent in peri-implantitis. Furthermore, local and controlled release using CHX chips aids in periodontal re-osseointegration; however, there are few clinical studies. Hence, further clinical studies on its application for bone re-osseointegration in peri-implantitis are needed.

CHX has specific disadvantages because clinically used $2 \%$ CHX permanently halts cell migration and significantly reduces fibroblast, myoblast, and osteoblast survival in vitro. ${ }^{42}$ Thus, further in vivo studies are required to examine and optimize CHX safety and efficacy.

\section{Antibiotics and Antimicrobial Agents}

Antibiotics are used in adjunct to mechanical therapy because they act against infection, either by inhibiting or killing the infectious agent. Different local and systemic antibiotic applications have been investigated. In peri-implantitis, the most commonly used local antibiotics are minocycline (MNO), doxycycline, gentamicin, and cefazolin. ${ }^{43-45}$
Local application of doxycycline or MNO following debridement and irrigating with an antiseptic agent is useful in treating moderately deep lesions. ${ }^{43}$ Cha et $\mathrm{al}^{44}$ evaluated the clinical, microbial, and radiographic effects of local MNO combined with surgical treatment of peri-implantitis. They found that repeated local delivery of MNO combined with surgical treatment provides increased clinical parameters and radiographic bone fill, with a higher treatment success rate in the short-term healing period. Furthermore, various polymeric films with antibiotics such as tetracycline hydrochloride polylactic acid, poly(e-caprolactone), and polymer/ tetracycline-containing solutions, reduce peri-implantitis development and associated pathogens. ${ }^{46}$ Local antibiotics, such as MNO, doxycycline, or CHX, are effectively combined with mechanical treatments for peri-implantitis, especially for incipient to moderate lesions. MNO and doxycycline have shown better results compared with CHX. Moreover, the combination of systemic antibiotics (such as ceftriaxone or gentamycin) and local antibiotics (tobramycin or gentamycin) demonstrate better treatment results.

Systemic antibiotic therapy increases the host defense to eliminate the infection by combating subgingival pathogens that remain following mechanical therapy. The combination of antibiotics (local and systemic) can be more beneficial in peri-implant infections. Furthermore, systemic antibiotic prophylaxis by injecting antibiotics at the lesion lowers the risk of postoperative infection. The surgical treatment of peri-implantitis can be combined with hydrogen peroxide and systemic antibiotics. A study found that the surgical treatment of peri-implantitis is effective and that therapy outcomes are affected by implant surface characteristics. ${ }^{47}$ However, the benefits of systemic antibiotics are not sustained for over 3 years.

Carcuac et $\mathrm{al}^{48}$ investigated the adjunctive use of systemic antibiotics and the local use of CHX for implant surface decontamination in peri-implantitis. They found that the treatment was successful in $45 \%$ of all implants but was 
higher in implants with an unmodified surface (79\%) compared with those with a modified surface (34\%). The local use of CHX had no overall effect on treatment outcomes. Although adjunctive systemic antibiotics had no impact on treatment success at implants with an unmodified surface, a positive effect on treatment success was observed at implants with a modified surface. There is a likelihood for treatment success, using adjunctive systemic antibiotics, in patients with implants with a modified surface; however, it was low. Hence, after careful assessment, it is recommended to use antibiotics to treat peri-implantitis.

Antimicrobial photodynamic therapy (aPDT) has emerged as a promising alternative to promote bacterial elimination and crestal bone remodeling in peri-implantitis. ${ }^{49}$ This technique is performed using direct mechanical debridement, followed by aPDT using $200 \mu \mathrm{g} / \mathrm{mL}$ methylene blue under red laser irradiation, which decontaminates the implant surface and surrounding tissue. Similarly, bioactive glass (BAG), especially Bioglass 45S5 and S53P4, are efficient antimicrobial agents, and their properties make BAG perhaps the ideal bone substitute for treating peri-implant infections..$^{50}$

\section{Surface Decontamination}

Nonsurgical mechanical therapy has a predictable outcome in peri-implant mucositis cases. However, it is more challenging when implant surfaces are exposed in peri-implantitis cases. Mechanical debridement alone may not completely remove the plaque because the instruments cannot access between the implant threads. ${ }^{51}$ Adjunctive treatments are proposed for surface decontamination to increase the efficiency of the nonsurgical treatment of peri-implant diseases.

\section{Chemical Methods}

Chemical methods include the local delivery of antibacterial agents. Commonly used chemicals for treating peri-implant diseases are described below.

\section{Citric Acid (CA)}

Although CA is used for cleaning implants, it is also the chemotherapeutic agent with the highest potential for removing the biofilm from contaminated Ti surfaces in vitro; however, it does not achieve complete removal..$^{52}$ Currently, the bactericidal effect of $\mathrm{CA}$ against biofilms has not been investigated on Ti surfaces. Burnishing with $\mathrm{CA}(\mathrm{pH}=1)$ for 1 minute significantly decreased the amount of E. coli lipopolysaccharide (LPS) on grit-blasted Ti alloy surfaces. ${ }^{35}$ Gosau et a ${ }^{53}$ performed a clinical study to evaluate the efficacy of six antimicrobial agents, sodium hypochlorite $(\mathrm{NaOCl})$, Hydrogen peroxide (HP) 3\%, CHX 0.2\%, Plax (triclosan), Listerine, and $40 \%$ citric acid on the surface decontamination of an oral biofilm attached to titanium implants. They found that the total bacterial load on the Ti surfaces was significantly higher in the control solution, phosphate-buffered saline, after incubation compared with the antiseptic groups. Hence, all tested antiseptics reduced microorganisms accumulated on the Ti surfaces. Moreover, CA and Plax had a significantly lower bactericidal effect against bacteria compared with $\mathrm{NaOCl}, \mathrm{HP}$, CHX, and Listerine.
CA toxicity has been investigated. CA at 4 to $10 \%$ concentrations did not demonstrate toxic effects on human osteoblasts. $^{54}$ In contrast, $40 \% \mathrm{CA}$ (pH 1) for 30 to 60 seconds may have a toxic effect on the peri-implant tissues and the implant and abutment junction due to its acidic pH. Hence, we need more clinical studies on how to effectively apply CA in order to avoid tissue contact.

\section{Ethyldiaminetetraacetic Acid (EDTA)}

The use of EDTA in dentistry is primarily as a chelating agent to eliminate the smear layer for periodontal regeneration and peri-implantitis. Wohlfahrt et al ${ }^{55}$ debrided 32 peri-implantitis defects with Ti curettes, cleaned them with $24 \%$ EDTA for 2 minutes, and rinsed them with saline and found that the EDTA reduced the PD by $2.6 \mathrm{~mm}$. Furthermore, Kotsakis et al $1^{56}$ treated implant surfaces with $20 \%$ CA, $0.12 \%$ CHX, 24\% EDTA, $1.5 \%$ sodium hydrochloride, or sterile saline (control). Their results demonstrated that the bacterial counts were significantly reduced after the decontamination and use of the chemotherapeutic agents. However, the agent residue caused some cytotoxic effects compared with control. Thus, EDTA should be used for treating peri-implantitis with caution.

\section{Hydrogen Peroxide (HP)}

Hydrogen peroxide is effective in decreasing the number of bacteria and fungi, for example, C. albicans, S. sanguinis or S. epidermidis from Ti specimens. ${ }^{57}$ Rubbing implants with $3 \%$ HP for 1 minute significantly decreased the E. Coli LPS from gritblasted Ti alloy and HA-coated strips versus the untreated samples. ${ }^{35}$ Similarly, another study found that $10 \% \mathrm{HP}$ inactivated the human biofilm and removed $99.9 \%$ of the bacteria from the implant surface..$^{22}$ Moreover, 10\% HP (swabbing for 1 minute) can also be used to clean the implant surface, which resulted in re-osseointegration in peri-implantitis lesions. However, $\mathrm{HP}$ is extremely reactive and may harm oral tissues if they are exposed to high-strength HP for a prolonged duration. Thus, HP should be used in treating peri-implantitis with caution.

\section{Saline}

Cleaning the implant surface with curettes and saline generates clinically stable results in peri-implantitis. Surgically debriding the implant with curettes, followed by rinsing with sterile saline and postoperative antibiotics (amoxicillin and metronidazole) prevents the advancement of peri-implantitis. A study found that postoperative amoxicillin resulted in an increased number of resistant anaerobes and a decreased number of sensitive facultative bacteria and facultative Gram-positive cocci compared with placebo; however, there were no signs/symptoms of infection in any group..$^{58}$

The use of adjunct chemical agents may improve the ability of saline to decontaminate the implant surface. However, a significant clinical benefit has not been demonstrated. Currently, there are still no conclusive studies showing the benefit of any of these agents compared with the other.

\section{Simulated Radiation Emission (Lasers)}

Stimulated radiation emission has demonstrated a beneficial therapeutic effect in peri-implantitis and can be used to 
support conventional mechanical therapy. ${ }^{59}$ The advantages of laser treatment include patient comfort, pain relief, and better results for specific applications. ${ }^{60}$ The various lasers investigated for treating peri-implantitis are erbium-doped yttrium aluminum garnet (Er:YAG) laser, diode laser, and carbon dioxide $\left(\mathrm{CO}_{2}\right)$ laser. ${ }^{61}$ Laser therapy, in combination with nonsurgical or surgical therapy for treating peri-implant diseases, provided minimal benefit in PD reduction, clinical attachment level gain, recession reduction, and plaque index reduction..$^{62}$ Lasers, when used as an adjunct to nonsurgical therapy, might result in more BOP reduction over the short-term.

Laser application in peri-implant areas results in the activation of cellular photoreceptors (cytochrome $\mathrm{C}$ oxidase) absorbing the laser radiation and delivers it to the cell's mitochondria. This increases the cell's adenosine triphosphate, which is the product of cytochrome $\mathrm{C}$ oxidase and the Krebs cycle, level, and increases cellular activity. ${ }^{63}$ Increased adenosine triphosphate stimulates macrophages, fibroblasts, mast cells, endothelial cells, bradykinin, nerve cells, and growth factors, which increase collagen synthesis, resulting in tissue regeneration.

The Er:YAG laser settings used in treating peri-implantitis are $100 \mathrm{~mJ} /$ pulse, $1 \mathrm{~W}, 10 \mathrm{~Hz}$, and $12.74 \mathrm{~J} / \mathrm{cm}^{2}$ for 60 seconds. ${ }^{64}$ Care should be used when using an Er:YAG laser $2940 \mathrm{~nm}$ wavelength to avoid adverse thermal effects on the implant surface. In a clinical study, Clem and Gunsolley ${ }^{65}$ evaluated the effective treatment regime for peri-implantitis lesions with deep ( $\geq 6 \mathrm{~mm}$ ) defects using an Er:YAG laser for implant surface decontamination, removing defect granulomatous tissues, and grafting therapy for bony defect resolution. They found that the mean PD was reduced by approximately $3.5 \mathrm{~mm}$ at 12 months and remained stable (mean $3.2 \mathrm{~mm}$ 12 months later). Radiographically, PDs were reduced due to peri-implant defect bone fill. Similarly, Yoshino et al ${ }^{66}$ found that antibiotic therapy significantly reduced the bacterial amount from the peri-implantitis sites and that Er:YAG laser therapy, along with bone augmentation, enhanced bone regeneration in the peri-implant bone defects.

The use of a low-intensity diode laser increased soft-tissue regeneration. Pai et $\mathrm{al}^{67}$ found that the clinical benefits of the laser supported other peri-implantitis treatments in their case series. They demonstrated that a diode laser had positive effects when treating peri-implantitis and dental implant osseointegration. Furthermore, a systematic review showed that laser use resulted in similar PD reduction compared with conventional mechanical debridement in the short-term. ${ }^{68}$ In contrast, Kotsakis et al ${ }^{69}$ recommended that laser therapy in peri-implantitis should be used as a phase I therapy. Moreover, a combination of nonsurgical treatment using granulation tissue curettage, laser detoxification, CHX irrigation, and MNO ointment injection resulted in bone formation..$^{70}$ Hence, a combination treatment is essential for an effective outcome.

Similarly, $\mathrm{CO}_{2}$ lasers can be used to treat peri-implantitis. ${ }^{61}$ Continuous wet $\mathrm{CO}_{2}$ lasers are more effective compared with dry $\mathrm{CO}_{2}$ laser in treating peri-implantitis. Because the clinical outcomes from $\mathrm{CO}_{2}$ lasers are unstable, these lasers are less commonly used and investigated. To determine its clinical effectiveness, further clinical trials should be performed.

These results indicate that peri-implantitis can be treated effectively using lasers with no damage to the surrounding tissues, but they also suggest that further investigations are required to determine the clinical efficacy of laser treatment. In addition, future research should focus on different types of lasers in clinical studies and long-term clinical outcome.

\section{Surgical Treatment}

Surgical treatment allows access to clean the inflammatory lesion in peri-implantitis. Surgical intervention therapy is recommended for treating peri-implantitis for a more favorable outcome. Surgical treatment includes access flap and debridement, access flap and bone recontouring or resective surgery, and regenerative approaches using bone grafts with or without a membrane. ${ }^{51}$ Implant surface decontamination is critical and often performed. Incomplete surface debridement can obstruct bone regeneration on the previously exposed surface of diseased implants. Surface decontamination can be achieved by various modalities, as previously mentioned.

Jepsen et $\mathrm{al}^{71}$ compared the effects of surgical treatment of peri-implant defects between using open flap debridement (OFD) and OFD plus porous titanium granules (PTGs). The OFD plus PTG group demonstrated a mean reduction in PD of $2.8 \mathrm{~mm}$ compared with $2.6 \mathrm{~mm}$ in the OFD group. BOP reduced from 89.4 to $33.3 \%$ and from 85.8 to $40.4 \%$ for the test and control groups, respectively. Besides, there was no significant difference in the complete resolution of peri-implantitis because this was achieved in $30 \%$ of the implants in the test group and $23 \%$ of the implants in the control group. Reconstructive surgery using PTGs resulted in significantly enhanced radiographic defect bone fill compared with OFD.

If nonsurgical treatment for peri-implantitis fails or if the peri-implant disease is at the moderate or severe stage, surgical therapy can be considered. Surgical correction of the PD and bone recontouring with plaque control is important in active peri-implant disease.

\section{Air-abrasive Powder (AP)}

AP uses an abrasive powder of sodium bicarbonate, calcium phosphate, or the amino acid glycine, which is driven by compressed air to eliminate the biofilm. ${ }^{72,73}$ AP treatment efficiently cleans contaminated implant surfaces. ${ }^{73,74}$

Tastepe et $\mathrm{al}^{75}$ evaluated AP treatment as an implant surface cleaning method for peri-implantitis. They found that considerable re-osseointegration (39-46\%) was achieved with improved clinical parameters after treatment when used in combination with surgical treatment. The treatment results are influenced by the powder type used, the application time, and whether the powder was applied surgically or nonsurgically. They concluded that the in vivo data on AP treatment as an implant surface cleaning method is insufficient to draw definitive conclusions. These results were similar to the results obtained by Schwartz et al, ${ }^{74}$ who found that glycine AP was effective in treating mucositis, enhanced the efficiency of nonsurgical treatment, and resulted in partial 
bone regeneration. Hence, clinicians can consider using AP for implant surface cleaning in peri-implantitis treatment.

Debridement with air abrasion facilitates the mechanical removal of bacterial biofilms but may damage implant surfaces on a microscopic level. Matsubara et $\mathrm{al}^{72}$ investigated the cleaning potential of various APs and their effect on titanium implant surfaces. They used three types of APs: sodium bicarbonate, glycine, and erythritol for 60 seconds. They found significant differences in cleaning potential between the groups. Sodium bicarbonate was the only powder that significantly increased implant roughness on the implant collar $(1.53-2.10 \mu \mathrm{m})$ and threads $(3.53-4.20 \mu \mathrm{m})$. Although the large-sized powder resulted in the greatest cleaning effect, it also caused more alterations on the implant surface. Glycine and erythritol treatment displayed no significant changes in surface roughness; however, they demonstrated a limited ink removal capacity.

\section{Resective Surgery}

The objective of resective surgery is to reduce pocket depth utilizing osteoplasty and/or ostectomy, correct the osseous defect, and allow better flap adaptation. In addition to bone recontouring, implantoplasty (smoothening) of the implant surface can be performed. An apically positioned flap combined with osteoplasty and implantoplasty was effective and reliable in treating peri-implantitis; however, an increased gingival recession may limit its use in esthetic areas. $^{76}$

\section{Implantoplasty}

Implantoplasty, also known as fixture modification, is the process of removing an infected, exposed implant surface. The goals of implantoplasty are to decontaminate the infected implant surface and gain a smooth surface, which decreases plaque adherence. ${ }^{77}$ Implantoplasty is usually performed together with resective osseous surgery and an apically positioned flap. This procedure can be performed using a high-speed diamond burr to remove the implant threads, followed by an Arkansas burr to polish the surface. These burrs were found to be the most effective in generating the smoothest implant surface. ${ }^{78}$ Implantoplasty combined with ostectomy and osteoplasty is effective in eliminating the progression of peri-implantitis. These results indicate that pocket removal with bone recontouring and plaque control is effective in treating peri-implantitis.

The disadvantage of implantoplasty is the metal debris, which is generally cytotoxic and genotoxic, from implants that often remain in the peri-implant tissues, which may cause adverse effects. The physicochemical properties and concentration of the debris determine the degree of the harmful effects. These effects can be prevented using a rubber dam or bone wax to protect the soft tissue during the procedure. Implantoplasty can also weaken the implant-abutment complex, especially in an implant less than $3.75 \mathrm{~mm}$ in diameter. A study found that implantoplasty significantly reduced the bending strength of narrow implants, with no effect in wide implants. ${ }^{79}$ Hence, implantoplasty should be done with caution on narrow implants and single implants subjected to more occlusal load.

\section{Regenerative Surgery}

The regenerative approach attempts to regenerate bone around peri-implantitis sites. The materials used are bone grafts, with or without membranes, or membrane alone. Biologic agents, such as growth factors or bone morphogenic proteins, can be considered. ${ }^{2}$

Various types of graft materials with or without a collagen membrane are often used for bone regeneration and bone augmentation. The bone graft acts as a scaffold; thus, it may improve bone regeneration because the barrier membrane maintains the space for cell infiltration and should be considered, especially in large defects. However, previous studies did not demonstrate clinical benefits of using a membrane. A systematic review demonstrated that regenerative treatment led to a $1.97 \mathrm{~mm}$ radiographic bone gain, $2.78 \mathrm{~mm}$ PD reduction, and 55\% decrease in the BOP. Using membranes and submerged healing did not improve the clinical parameters. ${ }^{80}$ Wiltfang et al ${ }^{81}$ evaluated regenerative treatment using autologous and xenogeneic bone graft with growth factors and found a reduced PD of $4 \mathrm{~mm}$ at the 1-year follow-up.

Various dental soft and hard tissues have been successfully regenerated in vitro using stem cells, indicating promising advancement in tissue engineering in dentistry. ${ }^{82-84}$ Growth factors and stem cell therapies are also being used for tissue regeneration in peri-implantitis cases. A study found that the recombinant human platelet-derived growth factor resulted in a higher percentage (40\%) bone fill, due to its osteoconductive property, which subsequently increased the clinical attachment level compared with $\beta$-tricalcium phosphate ( $\beta$-TCP)..$^{85}$ Acemannan sponges (Aloe vera extract) have generated bone formation in bone defects and may be used in peri-implantitis; however, further clinical studies are needed. ${ }^{86}$

A bone graft with a membrane may facilitate space maintenance favoring bone regeneration. ${ }^{87}$ Thus, this approach may provide the most favorable outcome. However, evidence has shown that the regenerative approach for treating a peri-implant defect remains unpredictable. Partial regeneration is possible in implant defects using various graft materials with resorbable membranes after guided bone regeneration. However, nonresorbable membranes have a disadvantage in that they must be removed by performing another surgery.

Surgical treatment may not be a good option in some peri-implantitis cases. When there is a substantial loss of bone in peri-implantitis (half the length of the implant), the success of surgery is unlikely..$^{88}$ Implants that are placed in an improper position can limit treatment outcomes. Additionally, implant mobility indicates advanced bone loss (>60\%) or a lack of osseointegration of the implant. In these cases, implant removal is recommended. ${ }^{89}$ If implant removal is necessary, then the second implantation should be performed with a larger diameter implant. ${ }^{90}$ 


\section{Conclusion}

Peri-implantitis is a common problem resulting in tissue destruction and implant loss. Plaque accumulation and biofilm formation play a major role in its initiation and development. Prosthetic factors such as residual cement and overloading may result in peri-implantitis but need clinical evidence. Routine supportive therapy reduces the risk of the onset of peri-implantitis. The peri-implantitis treatment modality should be selected based on the extent of disease. Nonsurgical mechanical therapy is beneficial and should be the initial treatment. The use of antibiotics limited impact on treatment success in peri-implantitis. Lasers remove the early supragingival biofilm and using a low-intensity laser induces soft-tissue regeneration. Implantoplasty is performed to smoothen the implant surface using rotary instruments. Various regenerative treatments have shown partial regeneration, but achieving total reosseointegration is difficult.

\section{Note}

The manuscript has not been published or submitted elsewhere. The manuscript has been read and approved by all the authors.

\section{Conflict of Interest}

None declared.

\section{References}

1 Amornvit P, Rokaya D, Bajracharya S, Keawcharoen K, Supavanich W. Management of obstructive sleep apnea with implant retained mandibular advancement device. World J Dent 2014;5(3):184-189

2 Moraschini V, Poubel LA, Ferreira VF, Barboza EdosS. Evaluation of survival and success rates of dental implants reported in longitudinal studies with a follow-up period of at least 10 years: a systematic review. Int J Oral Maxillofac Surg 2015;44(3):377-388

3 Lee CT, Huang YW, Zhu L, Weltman R. Prevalences of peri-implantitis and peri-implant mucositis: systematic review and meta-analysis. J Dent 2017;62:1-12

4 Berglundh T, Armitage G, Araujo MG, et al. Peri-implant diseases and conditions: consensus report of workgroup 4 of the 2017 World Workshop on the Classification of Periodontal and Peri-Implant Diseases and Conditions. J Clin Periodontol 2018;45(Suppl 20) :S286-S291

5 Najeeb S, Zafar MS, Khurshid Z, Zohaib S, Almas K. The role of nutrition in periodontal health: an update. Nutrients 2016;8(9):530

6 Niazi F, Naseem M, Khurshid Z, Zafar MS, Almas K. Role of Salvadora persica chewing stick (miswak): a natural toothbrush for holistic oral health. Eur J Dent 2016;10(2):301-308

7 Matsuda S, Movila A, Suzuki M, et al. A novel method of sampling gingival crevicular fluid from a mouse model of periodontitis. J Immunol Methods 2016;438:21-25

8 Carcuac $\mathrm{O}$, Berglundh T. Composition of human peri-implantitis and periodontitis lesions. J Dent Res 2014;93(11):1083-1088

9 Zhang L, Li X, Yan H, Huang L. Salivary matrix metalloproteinase (MMP)-8 as a biomarker for periodontitis: a PRISMAcompliant systematic review and meta-analysis. Medicine (Baltimore) 2018;97(3):e9642

10 Papi P, Di Carlo S, Rosella D, De Angelis F, Capogreco M, Pompa G. Peri-implantitis and extracellular matrix antibodies: a case-control study. Eur J Dent 2017;11(3):340-344
11 Teles R, Teles F, Frias-Lopez J, Paster B, Haffajee A. Lessons learned and unlearned in periodontal microbiology. Periodontol 2000 2013;62(1):95-162

12 Hasturk H, Kantarci A. Activation and resolution of periodontal inflammation and its systemic impact. Periodontol 2000 2015;69(1):255-273

13 Renvert S, Polyzois I. Risk indicators for peri-implant mucositis: a systematic literature review. J Clin Periodontol 2015;42(Suppl 16) :S172-S186

14 Staubli N, Walter C, Schmidt JC, Weiger R, Zitzmann NU. Excess cement and the risk of peri-implant disease - a systematic review. Clin Oral Implants Res 2017;28(10):1278-1290

15 Katafuchi M, Weinstein BF, Leroux BG, Chen YW, Daubert DM. Restoration contour is a risk indicator for peri-implantitis: a cross-sectional radiographic analysis. J Clin Periodontol 2018;45(2):225-232

16 Jepsen S, Berglundh T, Genco R, et al. Primary prevention of peri-implantitis: managing peri-implant mucositis. J Clin Periodontol 2015;42(Suppl 16) :S152-S157

17 Schwarz F, Derks J, Monje A, Wang HL. Peri-implantitis. J Clin Periodontol 2018;45(Suppl 20) :S246-S266

18 Peri-implant mucositis and peri-implantitis: a current understanding of their diagnoses and clinical implications. J Periodontol 2013;84(4):436-443

19 Sasada Y, Cochran DL. Implant-abutment connections: a review of biologic consequences and peri-implantitis implications. Int J Oral Maxillofac Implants 2017;32(6):1296-1307

20 Schwarz F, Alcoforado G, Nelson K, et al. Impact of implant-abutment connection, positioning of the machined collar/microgap, and platform switching on crestal bone level changes. Camlog Foundation Consensus Report. Clin Oral Implants Res 2014;25(11):1301-1303

21 Monje A, Insua A, Wang H-L. Understanding peri-implantitis as a plaque-associated and site-specific entity: on the local predisposing factors. J Clin Med 2019;8(2):279

22 Krennmair G, Seemann R, Piehslinger E. Dental implants in patients with rheumatoid arthritis: clinical outcome and peri-implant findings. J Clin Periodontol 2010;37(10): 928-936

23 Korsch M, Obst U, Walther W. Cement-associated peri-implantitis: a retrospective clinical observational study of fixed implant-supported restorations using a methacrylate cement. Clin Oral Implants Res 2014;25(7):797-802

24 Yi Y, Koo KT, Schwarz F. Ben Amara H, Heo SJ. Association of prosthetic features and peri-implantitis: a cross-sectional study. J Clin Periodontol 2020;47(3):392-403

25 Goiato MC, Shibayama R, Gennari Filho H, et al. Stress distribution in implant-supported prostheses using different connection systems and cantilever lengths: digital photoelasticity. J Med Eng Technol 2016;40(2):35-42

26 Amornvit P, Rokaya D, Keawcharoen K, Thongpulsawasdi N. Stress distribution in implant retained finger prosthesis: a finite element study. J Clin Diagn Res 2013;7(12):2851-2854

27 Chang M, Chronopoulos V, Mattheos N. Impact of excessive occlusal load on successfully-osseointegrated dental implants: a literature review. J Investig Clin Dent 2013;4(3):142-150

28 Sheridan RA, Decker AM, Plonka AB, Wang HL. The role of occlusion in implant therapy: a comprehensive updated review. Implant Dent 2016;25(6):829-838

29 Renvert S, Persson GR, Pirih FQ, Camargo PM. Peri-implant health, peri-implant mucositis, and peri-implantitis: case definitions and diagnostic considerations. J Clin Periodontol 2018;45(Suppl 20) :S278-S285

30 Costa FO, Takenaka-Martinez S, Cota LO, Ferreira SD, Silva GL, Costa JE. Peri-implant disease in subjects with and without preventive maintenance: a 5-year follow-up. J Clin Periodontol 2012;39(2):173-181 
31 Monje A, Aranda L, Diaz KT, et al. Impact of maintenance therapy for the prevention of peri-implant diseases: a systematic review and meta-analysis. J Dent Res 2016;95(4):372-379

32 Farsai PS. Supportive therapy (SPT) can potentially improve implant survival rate (sr), peri-implantitis, and peri-implant mucositis. J Evid Based Dent Pract 2020;20(1):101414

33 Ephros H, Kim S, DeFalco R. Peri-implantitis: evaluation and management. Dent Clin North Am 2020;64(2):305-313

34 Toma S, Brecx MC, Lasserre JF. Clinical evaluation of three surgical modalities in the treatment of peri-implantitis: a randomized controlled clinical trial. J Clin Med 2019;8(7):E966

35 Valderrama P, Wilson TG Jr. Detoxification of implant surfaces affected by peri-implant disease: an overview of surgical methods. Int J Dent 2013;2013:740680

36 Zafar MS. Comparing the effects of manual and ultrasonic instrumentation on root surface mechanical properties. Eur J Dent 2016;10(4):517-521

37 Persson GR, Samuelsson E, Lindahl C, Renvert S. Mechanical non-surgical treatment of peri-implantitis: a single-blinded randomized longitudinal clinical study. II. Microbiological results. J Clin Periodontol 2010;37(6):563-573

38 Pedrazzi V, Escobar EC, Cortelli JR, et al. Antimicrobial mouthrinse use as an adjunct method in peri-implant biofilm control. Braz Oral Res 2014;28(Spec No) :S1806-83242014000200301

39 Hallström H, Persson GR, Lindgren S, Renvert S. Open flap debridement of peri-implantitis with or without adjunctive systemic antibiotics: a randomized clinical trial. J Clin Periodontol 2017;44(12):1285-1293

40 Rudolf J-L, Moser C, Sculean A, Eick S. In-vitro antibiofilm activity of chlorhexidine digluconate on polylactide-based and collagen-based membranes. BMC Oral Health 2019;19(1):291

41 Menezes KM, Fernandes-Costa AN, Silva-Neto RD, Calderon PS, Gurgel BC. Efficacy of $0.12 \%$ chlorhexidine gluconate for non-surgical treatment of peri-implant mucositis. J Periodontol 2016;87(11):1305-1313

42 Liu JX, Werner J, Kirsch T, Zuckerman JD, Virk MS. Cytotoxicity evaluation of chlorhexidine gluconate on human fibroblasts, myoblasts, and osteoblasts. J Bone Jt Infect 2018;3(4):165-172

43 van Winkelhoff AJ. Antibiotics in the treatment of peri-implantitis. Eur J Oral Implantology 2012;5(Suppl):S43-S50

44 Cha JK, Lee JS, Kim CS. Surgical therapy of peri-implantitis with local minocycline: a 6-month randomized controlled clinical trial. J Dent Res 2019;98(3):288-295

45 Heo S, Kim H-J, Joo J-Y, Lee J, Kim S-J, Choi J. Simplified nonsurgical treatment of peri-implantitis using chlorhexidine and minocycline hydrochloride. J Periodontal Implant Sci 2018;48(5):326-333

46 Rokaya D, Srimaneepong V, Sapkota J, Qin J, Siraleartmukul K, Siriwongrungson V. Polymeric materials and films in dentistry: an overview. J Adv Res 2018;14:25-34

47 Carcuac O, Derks J, Abrahamsson I, Wennström JL, Petzold M, Berglundh T. Surgical treatment of peri-implantitis: 3-year results from a randomized controlled clinical trial. J Clin Periodontol 2017;44(12):1294-1303

48 Carcuac O, Derks J, Charalampakis G, Abrahamsson I, Wennström J, Berglundh T. Adjunctive systemic and local antimicrobial therapy in the surgical treatment of peri-implantitis: a randomized controlled clinical trial. J Dent Res 2016; 95(1):50-57

49 Garcia de Carvalho G, Sanchez-Puetate JC, Casalle N, Marcantonio E, Jr, Leal Zandim-Barcelos D. Antimicrobial photodynamic therapy associated with bone regeneration for peri-implantitis treatment: A case report. Photodiagn Photodyn Ther 2020;30:101705

50 Skallevold HE, Rokaya D, Khurshid Z, Zafar MS. Bioactive glass applications in dentistry. Int J Mol Sci 2019;20(23):E5960

51 Machtei EE. Treatment alternatives to negotiate peri-implantitis. Adv Med 2014;2014:487903
52 Ntrouka VI, SlotDE, Louropoulou A, Van der Weijden F. The effect of chemotherapeutic agents on contaminated titanium surfaces: a systematic review. Clin Oral Implants Res 2011;22(7): 681-690

53 Gosau M, Hahnel S, Schwarz F, Gerlach T, Reichert TE, Bürgers R. Effect of six different peri-implantitis disinfection methods on in vivo human oral biofilm. Clin Oral Implants Res 2010;21(8):866-872

54 Guimarães LF, Fidalgo TK, Menezes GC, Primo LG, Costa e Silva-Filho F. Effects of citric acid on cultured human osteoblastic cells. Oral Surg Oral Med Oral Pathol Oral Radiol Endod 2010;110(5):665-669

55 Wohlfahrt JC, Lyngstadaas SP, Rønold HJ, et al. Porous titanium granules in the surgical treatment of peri-implant osseous defects: a randomized clinical trial. Int J Oral Maxillofac Implants 2012;27(2):401-410

56 Kotsakis GA, Lan C, Barbosa J, et al. Antimicrobial agents used in the treatment of peri-implantitis alter the physicochemistry and cytocompatibility of titanium surfaces. J Periodontol 2016;87(7):809-819

57 Bürgers R, Witecy C, Hahnel S, Gosau M. The effect of various topical peri-implantitis antiseptics on Staphylococcus epidermidis, Candida albicans, and Streptococcus sanguinis. Arch Oral Biol 2012;57(7):940-947

58 Moslemi N, Shahnaz A, Bahador A, Torabi S, Jabbari S, Oskouei ZA. Effect of postoperative amoxicillin on early bacterial colonization of peri-implant sulcus: a randomized controlled clinical trial. J Dent (Tehran) 2016;13(5):309-317

59 Alshehri FA. The role of lasers in the treatment of peri-implant diseases: a review. Saudi Dent J 2016;28(3):103-108

60 Najeeb S, Khurshid Z, Zafar MS, Ajlal S. Applications of light amplification by stimulated emission of radiation (lasers) for restorative dentistry. Med Princ Pract 2016;25(3):201-211

61 Ashnagar S, Nowzari H, Nokhbatolfoghahaei H. Yaghoub Zadeh B, Chiniforush N, Choukhachi Zadeh N. Laser treatment of peri-implantitis: a literature review. J Lasers Med Sci 2014;5(4):153-162

62 Lin G-H, Suárez López Del Amo F, Wang H-L. Laser therapy for treatment of peri-implant mucositis and peri-implantitis: An American Academy of Periodontology best evidence review. J Periodontol 2018;89(7):766-782

63 Mehdiyev I, Gülsen U, Sentürk MF, Sayan NB. Radiographic evaluation of low-level laser therapy-enhanced maxillary sinus augmentation with simultaneous dental implant placement. Ann Maxillofac Surg 2019;9(1):48-52

64 Smeo K, Nasher R, Gutknecht N. Antibacterial effect of Er:YAG laser in the treatment of peri-implantitis and their effect on implant surfaces: a literature review. Laser Dent Sci 2018;2(4):201-211

65 Clem D, Gunsolley JC. Peri-implantitis treatment using Er:YAG laser and bone grafting. a prospective consecutive case series evaluation: 1 year posttherapy. Int J Periodontics Restorative Dent 2019;39(4):479-489

66 Yoshino T, Yamamoto A, Ono Y. Innovative regeneration technology to solve peri-implantitis by Er:YAG laser based on the microbiologic diagnosis: a case series. Int J Periodontics Restorative Dent 2015;35(1):67-73

67 Jagadish Pai B, Shridhar A, Kamath V, Jaiswal N, Malagi S, Radhika B. Adjunctive use of diode lasers in the treatment of peri-implantitis: a case series. J Dent Implant 2014;4:91-97

68 Mailoa J, Lin GH, Chan HL, MacEachern M, Wang HL. Clinical outcomes of using lasers for peri-implantitis surface detoxification: a systematic review and meta-analysis. J Periodontol 2014;85(9):1194-1202

69 Kotsakis GA, Konstantinidis I, Karoussis IK, Ma X, Chu H. Systematic review and meta-analysis of the effect of various laser wavelengths in the treatment of peri-implantitis. J Periodontol 2014;85(9):1203-1213 
70 Chang H-Y, Park S-Y, Kim J-A, Kim Y-K, Lee H-J. Early radiographic diagnosis of peri-implantitis enhances the outcome of peri-implantitis treatment: a 5-year retrospective study after non-surgical treatment. J Periodontal Implant Sci 2015;45(3):82-93

71 Jepsen K, Jepsen S, Laine ML, et al. Reconstruction of peri-implant osseous defects: a multicenter randomized trial. J Dent Res 2016;95(1):58-66

72 Matsubara VH, Leong BW, Leong MJL, Lawrence Z, Becker T, Quaranta A. Cleaning potential of different air abrasive powders and their impact on implant surface roughness. Clin Implant Dent Relat Res 2020;22(1):96-104

73 Tastepe CS, Liu Y, Visscher CM, Wismeijer D. Cleaning and modification of intraorally contaminated titanium discs with calcium phosphate powder abrasive treatment. Clin Oral Implants Res 2013;24(11):1238-1246

74 Schwarz F, Becker K, Renvert S. Efficacy of air polishing for the non-surgical treatment of peri-implant diseases: a systematic review. J Clin Periodontol 2015;42(10):951-959

75 Tastepe CS, van Waas R, Liu Y, Wismeijer D. Air powder abrasive treatment as an implant surface cleaning method: a literature review. Int J Oral Maxillofac Implants 2012;27(6):1461-1473

76 Englezos E, Cosyn J, Koole S. Jacquet W, De Bruyn H. Resective treatment of peri-implantitis: clinical and radiographic outcomes after 2 years. Int J Periodontics Restorative Dent 2018;38(5):729-735

77 Aljateeli M, Fu JH, Wang HL. Managing peri-implant bone loss: current understanding. Clin Implant Dent Relat Res 2012;14(Suppl 1) :e109-e118

78 Ramel CF, Lüssi A, Özcan M, Jung RE, Hämmerle $\mathrm{CH}$, Thoma DS. Surface roughness of dental implants and treatment time using six different implantoplasty procedures. Clin Oral Implants Res 2016;27(7):776-781

79 Chan HL, Oh WS, Ong HS, et al. Impact of implantoplasty on strength of the implant-abutment complex. Int J Oral Maxillofac Implants 2013;28(6):1530-1535

80 Daugela P, Cicciù M, Saulacic N. Surgical regenerative treatments for peri-implantitis: meta-analysis of recent findings in a systematic literature review. J Oral Maxillofac Res 2016;7(3):e15

81 Wiltfang J, Zernial O, Behrens E, Schlegel A, Warnke PH, Becker ST. Regenerative treatment of peri-implantitis bone defects with a combination of autologous bone and a demineralized xenogenic bone graft: a series of 36 defects. Clin Implant Dent Relat Res 2012;14(3):421-427

82 Zafar MS, Khurshid Z, Almas K. Oral tissue engineering progress and challenges. Tissue Eng Regen Med 2015;12(6):387-397

83 Sheikh Z, Najeeb S, Khurshid Z, Verma V, Rashid H, Glogauer M. Biodegradable materials for bone repair and tissue engineering applications. Materials (Basel) 2015;8(9):5744-5794

84 McGuire MK, Tavelli L, Feinberg SE, et al. Living cell-based regenerative medicine technologies for periodontal soft tissue augmentation. J Periodontol 2020;91(2):155-164

85 Darby IB, Morris KH. A systematic review of the use of growth factors in human periodontal regeneration. J Periodontol 2013;84(4):465-476

86 Trinh HA, Dam VV, Le B, Pittayapat P, Thunyakitpisal P. Indirect sinus augmentation with and without the addition of a biomaterial: a randomized controlled clinical trial. Implant Dent 2019;28(6):571-577

87 Sheikh Z, Hamdan N, Ikeda Y, Grynpas M, Ganss B, Glogauer M. Natural graft tissues and synthetic biomaterials for periodontal and alveolar bone reconstructive applications: a review. Biomater Res 2017;21:9

88 Decker AM, Sheridan R, Lin GH, Sutthiboonyapan P, Carroll W, Wang HL. A prognosis system for periimplant diseases. Implant Dent 2015;24(4):416-421

89 Hanif A, Qureshi S, Sheikh Z, Rashid H. Complications in implant dentistry. Eur J Dent 2017;11(1):135-140

90 Eroglu CN, Ertugrul AS, Eskitascioglu M, Eskitascioglu G. Changes in the surface of bone and acid-etched and sandblasted implants following implantation and removal. Eur J Dent 2016;10(1):77-81 